\title{
Global Beamforming and Demodulation For Linearly Modulated Signals
}

\author{
Josep Sala-Alvarez, Gregori Vázquez-Grau \\ Dpt Signal Theory and Communications \\ Polytechnic University of Catalonia, Apt 30002 \\ 08071 Barcelona, SPAIN \\ Tel: +34-3-4016440 / Fax: +34-3-4016447 \\ E-mail: alvarez@tsc.upc.es / gregori@tsc.upc.es
}

\begin{abstract}
The objective of this paper is to present a novel and efficient demodulation algorithm for arrayed receivers without explicit signal parameter estimation. Direct extraction of the digital information contained in the signal impinging on the array is adressed. It is shown that the algorithm implicitly performs globally tasks such as beamforming, equalization, timing recovery, etc. without training signals. The main goal is to force the pdf of the symbol estimates at the demodulator output to be as close as possible to the desired a priori pdf of the real symbols. That is, the algorithm uses a statistical reference in contrast to temporal or spatial reference.
\end{abstract}

\section{$1 \quad$ Introduction}

Several aspects differentiate arrayed demodulation from conventional, single-sensor demodulation. A suite of wellknown techniques are usually applied in the context of array signal processing for beam weight acquisition and tracking. Beamforming algorithms can be classified as non-parametric (MUSIC,Johnson,...) usually based on second order statistics of the received signal and adaptive methods based on the minimization of a cost function. Knowledge of an a priori known reference is used to acquire the optimum weights. Among them, we can mention TRB (temporal reference beamforming) or SRB (spatial reference beamforming)[Ref1]. Successful demodulation requires that those parameters that characterise the channel are estimated to a good degree of accuracy. The estimation of the complete set of parameters is usually decoupled to several algorithms. In some cases there exists a preference order in the estimation. Timing cannot be casily estimated unless the beamformer weights have converged to their optimum configuration. In this paper we will analyse the performance of a global demodulation algorithm. That is, one where all parameters of interest are inter-related through a cost function to be defined later on. The study will be restricted to recovery of linearly modulated signals (M-PSK, M-ASK, MQAM). The definition of the cost function as well as of the architccture are crutial points in the design. The objective will be to stecr the adaptive coefficients of the system so as to force a known pdf at the output (that of the symbol constcllation plus noise)[Ref2].

We will propose an architecture for global demodulation in arrayed receivers capable of performing the lasks of bcamforming. cqualisation and interpolation and clock and carrier phase recovery. The need for joint beamforming and equalisation appears in land mobile communications where antenna arrays are used as a means to ward off channel fading with space diversity techniques. The spatial distribution of the signal cannot be generally decomposed into a main beam and a few multipath rays, but due to the special propagation characteristics of the medium, diffuse multipath or Rayleigh fading usually occurs. In urban areas, signals are scattered and reflected by buildings, so that many rays impinge on the array from different directions and with different delays. Therefore, this can be modeled as passing the transmitted signal through a discrete-time single-input mltiple-output (SIMO) channel, making equalisation in the spatial and in the temporal domain necessary. It is then sought to spatially combine the sensor signals cooperatively with the equaliser. Antennas are spaced a number of wavelengths apart to guarantee independent fades and optimise diversity. Apperance of grating lobes is not so important as in this kind of application we cannot talk with propriety of a direction of arrival.

In Section 2 we will present the chosen architecture for global demodulation. It will also be discussed how synchronisaton and symbol interpolation can be incorporated into the equalization process. The chosen cost function and the related adaptive algorithm are presented in Section 3. It is explained how statistical reference can be taken to advantage in the choise of the appropriate cost function. Finally, simulations and conclusions are presented in Sections 4 and 5 respectively.

2

\section{Architecture}

The basic assumption for global demodulation is that a same architecture can perform all tasks necessary for the recovery of the modulated symbols from minimisation of a single cost function evaluated on the data. Here it will be shown that the synchronisation task can be roughly considered as a special case of adaptive equalisation, although it has some distinctive properties. Let us assume for clarity that the channel does not introduce any distortion and that the signal is only received with an unknown delay and carrier phase such that.

$$
r(n)=\sum_{k=-\infty}^{k=+\infty} a_{k} e^{j 0} p^{\prime}\left(n-k N_{s s}-\varepsilon\right)+n_{r}(n)
$$

with $r$ the received signal, $a_{k}$ the symbol sequence linearly modulated on a pulse $p^{\prime}(n)=p_{1}(n T)$, a discretised version of 
the time-continuous pulse $p_{t}, N_{s s}$ the number of samples per symbol, $\varepsilon$ the clock error betwen transmitter and receiver (such that $0 \leq \varepsilon \leq N_{S S}$ may be a non-integer) and $n_{I}(n)$ the received noise process. For the moment, as the carrier phase rotation is a memoryless operation on the transmitted signal, it will be of no concern to us. Let us also take the pulse $\mathrm{p}_{\mathrm{t}}$ to be the square root raised cosine pulse with roll-off $\alpha$, such that the symbol sequence can be obtained free of intersymbol interference by convolving the received sequence $I(n)$ with the filter matched to $p_{t}$ and sampling at the correct strobe. Let $h_{m f}(n)=p^{\prime}(-n)$ be the matched filter, then the output sequence is expressed as,

$$
\begin{aligned}
y(n) & =\sum_{k=-\infty}^{k=+\infty} h_{m f}(k) r(n-k) \\
& =\sum_{k=-\infty}^{k=+\infty} a_{k} e^{\theta} p_{y}^{\prime}\left(n-k N_{s s}-\varepsilon\right)+n_{y}(n)
\end{aligned}
$$

with $p_{y}^{\prime}(n)$ the equivalent pulse at the output of the matched filter with the property that $\mathrm{p}^{\prime} \mathrm{y}\left(\mathrm{mN}_{\mathrm{ss}}\right)=0$ and $\mathrm{n}_{\mathrm{y}}(\mathrm{n})$ is the corresponding filtered input noise sequence. We want then to synchronise to the input noise sequence by estimating the parameter $\varepsilon$. If $\varepsilon$ is an integer, one out of the $N_{s s}$ possible values will be free of intersymbol interference and it can be estimated by the usual methods (ML synchronisation)[Ref3]. Nevertheless, if $\varepsilon$ is a non-integer, sample interpolation must be carried out to obtain an estimate of the transmitted symbol. Interpolation can be performed with an adaptive filter placed at the output of the matched filter. The procedure is to impose a strobe at a given instant and let the adaptive filter evolve so as to eliminate interference. Therefore, if the strobe has not been correctly fixed at the actual symbol synchronisation, the filter coefficients will automatically converge to a delayed version of the pulse with respect to the strobe fixed by the demodulator. Obviously, when the channel introduces no ISI other methods will perform better, as for this case, misadjustment noise in the filter coefficients introduces residual intersymbol interference. Nevertheless, it has been shown that blind equalisation and sync recovery can be performed in a joint fashion by the same architecture.

There are several options to correct for carrier phase. We can either consider a complex equaliser instead of a real one, so that enough degrees of freedom are available to perform rotation of the constellation. In this case it would also be possible to correct for unbalance in the in-phase and quadrature channelisation unit due to differences in the antialiasing filters. In our approach we have chosen to encompass phase rotation in the complex beamforming weights. If the incoming signal displays a residual Doppler frequency, much smaller than the symbol rate, it can also be tracked in the beamforming stage.

\subsection{Beamforming and Equalisation}

Let us define the vector of discretised signals received at each sensor as $r(n)=r\left(n T_{s}\right)$, where $T_{s}$ stands for the sampling timc. Let us also define the data matrix $X_{n}=\mid r(n), r(n-$ 1),.., $r(n-N+1)$ ] as that containing the incoming sensor signals in a lime window of length $N$. The beamformer and equaliser coefficients are defined respectively by the vectors $w_{S}$ and $w_{t}$ for a narrow-band architecture or by $W$ for a wideband architecture. Hence, the demodulated symbols are obtained as,

$$
(N B A) y_{k}=\mathbf{w}_{s}^{H} \mathbf{X}_{k} \mathbf{w}_{1},(W B A) y_{k}=\operatorname{tr}\left(\mathbf{w}^{H} \mathbf{X}_{k}\right)
$$

where the output is obtained from a quadratic expression of the coefficients. Here only narrow-band architectures shall be considered.

\section{Cost Function}

As has already been stated, in this paper we will consider only blind signal recovery algorithms without any reference for initial training. The cost function of the adaptive algorithm is constructed in such a way that forces an output probability distribution as similar as possible to that of the desired symbol constellation of reference. We will show here two equivalent ways of deriving a suitable cost function. We want to maximise the probability that the symbol sequence at the output of the equaliser has been produced by a QPSK source. Therefore, we propose maximisation of the following expression for a general linear modulation of $\mathrm{M}_{0}$ levels,

$$
\begin{aligned}
& J=-\frac{1}{N_{J}} \sum_{k=0}^{k=N_{J}-1} \ln p_{a+n}\left(y_{k}\right) \\
& p_{a+n}\left(y_{k}\right)=\frac{1}{\pi \sigma^{2} M_{0}} \sum_{i^{\prime}=1}^{i^{\prime}=M_{0}} e^{-\left|y_{k}-a_{i}\right|^{2} / \sigma^{2}}
\end{aligned}
$$

where $a_{k}$ constinte the constellation symbols and $\sigma$ models the residual noise variance at the output of the equalizer. The cost function is minimised when all outputs of the equaliser $\hat{a}_{k}$ coincide with large probability with the maxima of the pdf of the constellation plus noise. When the equaliser weights have not converged yet, there appear spurious peaks in the actual pdf due to intersymbol interference that yield a higher value of the cost function. Convergence is reached when there is coincidence of the maxima of the actual pdf with those of the objective pdf. The noise variance is also minimised as sharper peaks in the actual pdf result in smaller values for the cost function. Once the cost function has been defined, we use the gradient rule to derive the adaptive algorithm. For simplicity we will assume that $\mathrm{NJ}_{\mathrm{J}}=1$, that is. a memoryless non-linearity at the output of the equaliser. Generalization is straightforward. Here the gradient must be calculated with respect to the equaliser and the beamformer weights as,

$$
\begin{aligned}
& \nabla_{\mathbf{w}_{1}} J=\left(\frac{d}{d y_{k}} J\right) \nabla_{\mathbf{w}_{1}} y_{k}=\varepsilon\left(y_{k}\right) \mathbf{w}_{s}^{\prime \prime} \mathbf{X} \\
& \nabla_{\mathbf{w}_{s}^{\prime \prime}} J=\varepsilon\left(y_{k}\right) \mathbf{X} \mathbf{w}_{1}
\end{aligned}
$$

where the generalised error function $\varepsilon\left(\hat{a}_{k}\right)$ is defined as the derivative of the cost function J. From equation [3.2], the cxact expression for $\mathcal{E}\left(\hat{a}_{k}\right)$ is found to bc, 


$$
\varepsilon\left(y_{k}\right)=\frac{e^{-J\left(y_{k}\right)}}{\pi \sigma^{4} M_{0}} \sum_{j^{\prime}=1}^{i^{\prime}=M_{0}} e^{-\left|x_{k}-a_{i}\right|^{2} / \sigma^{2}}\left(y_{k}-a_{i^{\prime}}\right)
$$

It can be easily observed that this is but a weighted average of the error of the received symbol with respect to all symbols in the constellation. The reliability we assign to each of the errors is controlled by the tentative noise variance $\sigma$. We can see that for very small values of $\sigma$, this error function can be interpreted as the error between the equaliser output $y_{k}$ and the symbol hard decision on this output. As $\sigma$ is assigned larger values, the algorithm is very conservative and assigns the error to the closest symbol a lesser weight in the averaging, increasing that of the rest of the errors.

The value of $\sigma$ is also adapted by the algorithm to match the actual spread of the constellation (a gradient update is used). During acquisition, $\sigma$ is initialised at a large value in order to robustize the likelihood of the decisions that generate the error sequence. Care must be taken so that the resulting cost function displays four distinct peaks (for a QPSK modulation). If $\sigma$ were chosen large enough so that the tails of any one peak mask the rest of the peaks, the algorithm would recover any Gaussian signal in the noise subspace instead of the intended communication signal. Therefore this variance is limited by setting $\sigma 2=\sigma_{0}{ }^{2}+\delta \sigma^{2}$. Only the parameter $\delta \sigma^{2}$ is adapted by the algorithm. Simulations can be observed in section 4 .

\subsection{Error Sequence}

We will seek here an equivalent way of deriving the expression in [3.3] for the error sequence of the adaptive algorithm. We need therefore to define an error sequence from which the adaptive weights can be driven. The conventional way in decision feedback equalisation is to perform a decision on the incoming symbol and reconstruct the error sequence through substraction of the estimated symbol from the incoming sample. In high noise situations erroneous decisions will degrade the performance of the adaptive algorithm as the actual correlation between the data and the estimated error is not zero. We propose here an algorithm for reconstructing the error sequence that is very conservative in terms of decision risk. The objective is to calculate all possible errors between the incoming sample and all constellation symbols and weighthem in accordance with an estimate of their likelihood. Let us assume that the incoming sample is $y_{k}$, then, the error sequence is constructed as

$$
\hat{\varepsilon}_{k}=\sum_{i=1}^{i=M_{0}} \hat{p}\left(a_{i} \mid y_{k}\right)\left(y_{k}-a_{i}\right)
$$

The set of estimated conditional probabilitics is obtained by assuming a tentative noise variance (which can also be updated by the adaptive algorithm). The actual expression for $p$ is given by application of the Baycs conditional probability rule with the actual noise variance,

$$
p\left(a_{i} \mid y_{k}\right)=p\left(y_{k} \mid a_{i}\right) p\left(a_{i}\right) / \sum_{i=1}^{i=M_{0}} p\left(y_{k} \mid a_{i}\right)
$$

The expression for the probability of $y_{k}$ conditioned on a given symbol is given straightforwardly by the following expression,

$$
p\left(y_{k} \mid a_{i}\right)=\left(\pi \sigma^{2}\right)^{-1} e^{-\left|y_{k}-a_{i}\right|^{2} / \sigma^{2}}
$$

If we assume equiprobability among all constellation symbols (that is, $\left.p\left(a_{i}\right)=1 / M_{0}\right)$, we arrive at the final expression for the tentative conditional probabilities of the symbols given the incoming samples. We have denoted the tentative noise variance as $\sigma_{\mathrm{t}}$,

$$
p\left(a_{i} \mid y_{k}\right)=M_{0}^{-1} e^{-\left|y_{k}-a_{i}\right|^{2} / \sigma_{i}^{2}} / \sum_{i^{\prime}=1}^{i=M_{0}} e^{-\left|y_{k}-a_{i}\right|^{2} / \sigma_{i}^{2}}
$$

Therefore, we obtain the same result for the error sequence as derived as the last section from minimisation of the cost function $\mathrm{J}$.

Note that when the noise power is small, $p\left(a_{i} l y_{k}\right)$ tends to be maximum for the tentative symbol close to the actual one. Other symbols are assigned comparatively smaller weights so that the corresponding errors have a small influence in the adaptation of the coefficients. During acquisition, when the beamformer or filter coefficients are far from optimal, the tentative probability distribution becomes uniform among symbols. Therefore, the errors are equally weighted. We can interpret this by considering that for errors much larger than the constellation dimensions, the constellation is comparatively reduced to a point as all errors have approximately the same magnitude. As the algorithm converges, the errors and the corresponding weighting factors become more and more differentiated.

\section{Simulations}

The fact that the architecture of the adaptive algorithm is as defined in equation [2.3] has important consequences on convergence. That is, the update of the equaliser coefficients and of the beamformer are tighly related with the gradient equations. Equalizer convergence is conditioned on beamformer convergence as it determines the SNR at the equalizer input. A scenario with one QPSK signals and two Gaussian signals impinging on a $\lambda / 25$-sensor linear array at different DOAs has been simulated. Sensor noise equally distributed in space has been superimposed at each sensor, with an SNR of $-1.1 \mathrm{~dB}$. The input signal is sampled at 4 samples per symbol. A number of 20 taps (5 symbols) has been chosen for the fractionally spaced equalizer. The beamformer is initialized with an omnidirectional pattern. The step size for the adaptation of the beamformer and equalizer coefficients is 0.005 and 0.01 for the update of the tentative output variance. $1 / \sigma^{2}$ has been initialized to 1.5 (and limited to a minimum of 1.25 to guarantec that two distinct peaks appear in the cost function). The incoming signal has becn filtered by a 7-tap mixed-phase channel. Simulations are depicted in figures 4.1 to 4.4 . 
The final radiation pattern is shown in figure 4.1. Note how the algorithm is able to discriminate the QPSK signal in front of the other two Gaussian sources without any reference. Figure 4.2 shows the evolution of the in-phase channel at one sample per symbol. The acquisition and tracking regimes are clearly differentiated. Final convergenge is quite fast once the array beam has been centered. The evolution of the estimated EbNo (dB) is depicted in Figure 4.3 (the inverse of the squared tentative variance divided by the number of bits in the constelation). Note that during acquisition the tentative variance is maximum and that the transition to tracking (at an estimated EbNo of $8 \mathrm{~dB}$ ) is rather fast. This is due to the masking effect of the beamformer. A side-effect of choosing an unconstrained adaptive $\sigma$ in the algorithm has been observed in high noise situations where $s$ grows sufficiently large to mask all peaks in the cost function. The net results is that the algorithm recovers a Gaussian signal instead of a discrete communications signal. Finally, in figure 4.4 we have depicted the EbNo curves at different background noise powers. A total of 30 averages has been performed at each SNR. The transition from acquisition to tracking appears smoother in contrast to figure 4.3 due to the random occurrence of the elbow. Note how acquisition takes longer at decreasing SNR.

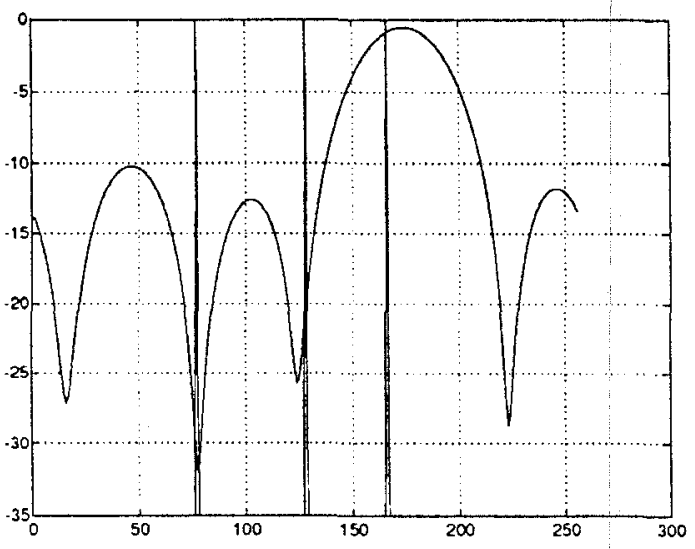

Fig. 4.1: final radiation pattern

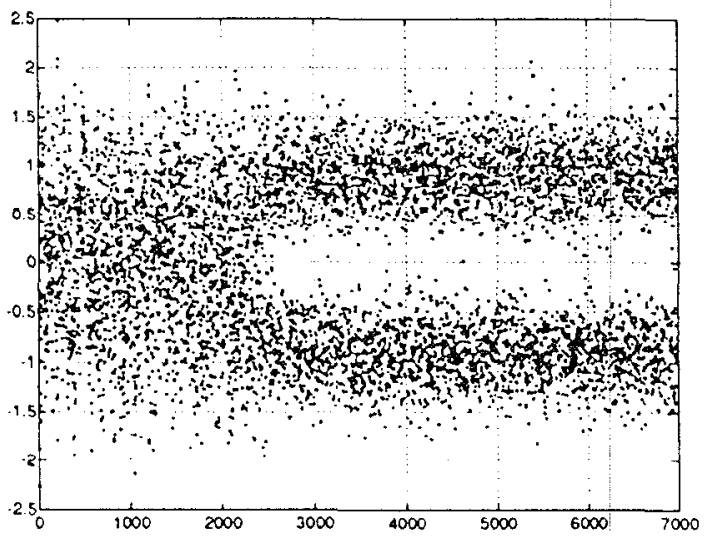

Fig. 4.2: cvolution of in-phase channel

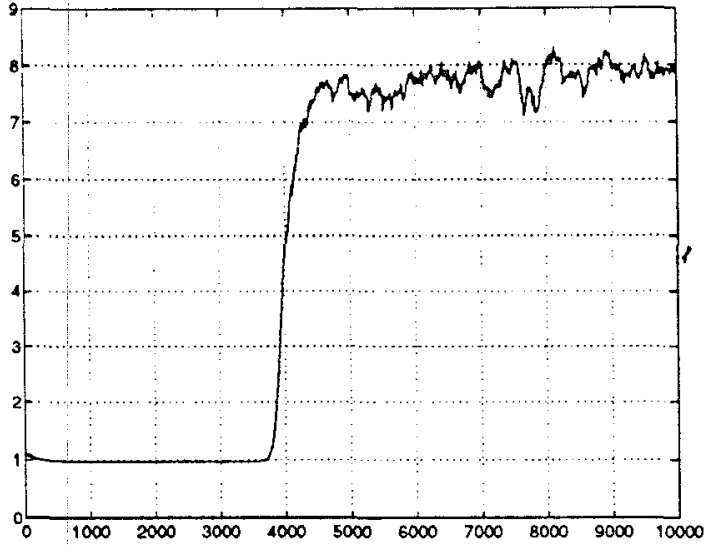

Fig. 4.3: Evolution of estimated EbNo.

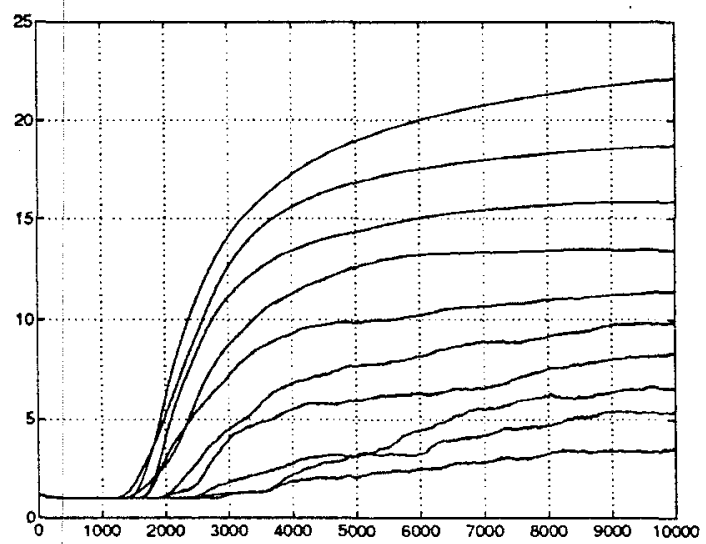

Fig. 4.4: evolution of estimated EbNo for several input SNR's: $16.8,10.8,7.3,4.8$, $2.8,1.3,0,-1.1,-2.2$ and $-3.1 \mathrm{~dB}$.

\section{5}

\section{Conclusions}

We have shown one blind algorithm capable of recovering signals of a given distribution with an arrayed demodulator thorugh careful dsign of a non-linearity. Carrier and timing acquisition, equalization and beamforming are performed successfully with the same algorithm. It has been shown that acquisition occurs in the range of a few thousand symbols which is fast for a blind algorithm. Further research is currently being done for the ease of recovering over one signal of the same statistics.

6

\section{References}

[1] Compton, R.T., "Adaptive Antennas, Concepts and Performance", Prentice-Hall, Inc, 1988.

[2] Sala, Josep;Vázquez, Gregori. "Adaptive Blind Equalization and Demodulation without Channcl and signal Parameter Extraction". Proceedings of EUSIPCO'94, Edinburgh, September 1994, Scotland. 